\title{
Prospective Audit and Feedback of Antimicrobial Stewardship in Critical Care: Program Implementation, Experience, and Challenges
}

\author{
Marion Elligsen, Sandra A N Walker, Andrew Simor, and Nick Daneman
}

\section{INTRODUCTION}

A ntimicrobial stewardship has been defined as the limitation of inappropriate use of antimicrobials while optimizing the selection, dosing, route, and duration of antimicrobial therapy to maximize clinical cure or prevent infection; limiting unintended consequences, such as emergence of resistance, occurrence of adverse drug events, and selection of pathogenic organisms (such as Clostridium difficile); and minimizing cost. ${ }^{1}$ Because of increased resistance to antimicrobial agents and a lack of novel agents, there has been increasing pressure for judicious use of currently available antimicrobials, to ensure that their activity is preserved. ${ }^{2}$ In Canada, the need for antimicrobial stewardship has been recognized as a patient safety issue by the Institute for Safe Medication Practices Canada, the Ontario Agency for Health Protection and Promotion, and the Ontario Ministry of Health and Long-Term Care, and together these agencies have launched the Ontario Antimicrobial Stewardship Project. ${ }^{3}$ Accreditation Canada has also proposed that it be a Required Organizational Practice for institutions to have a program to appropriately manage antimicrobial resources. ${ }^{4}$ However, despite the well-recognized need to institute antimicrobial stewardship programs, detailed descriptions of the steps taken and challenges encountered in implementing successful programs are lacking in the literature. The purpose of this article is to describe our experience with the development of an antimicrobial stewardship program at a university-affiliated teaching hospital.

Sunnybrook Health Sciences Centre (SHSC) is a tertiary care teaching hospital with 700 acute care beds located in Toronto, Ontario. Before 2007, this institution had an infectious diseases team (consisting of infectious diseases physicians and an infectious diseases pharmacist), which was committed to implementing institutional measures to optimize the use of antimicrobials. In addition, there was an active and engaged Antimicrobial Subcommittee. This subcommittee, which remains in existence at the time of writing, consists of the infectious diseases team and a drug information pharmacist. It reports to the hospital's Pharmacy and Therapeutics Committee, which in turn reports to the Medical Advisory Committee. Since 1993, the Antimicrobial Subcommittee had undertaken many antibiotic stewardship initiatives, including development of an antimicrobial handbook (updated annually), institutional antibiograms (also updated annually), provision of regular education about antimicrobials for pharmacists and medical residents, intermittent drug utilization audits, and development of policies for restricted antimicrobials, IV-to-oral step-down, and autosubstitution. In addition, the microbiology laboratory at this institution had adopted selective reporting of susceptibility results.

In January 2007, the Infectious Diseases Society of America published guidelines for antimicrobial stewardship. ${ }^{1}$ On the basis of these guidelines, the SHSC Antimicrobial Subcommittee concluded that the 2 primary deficiencies in the hospital's stewardship initiatives were the absence of prospective audit and feedback and the lack of a fully integrated database to enable impact assessment. It was recognized that implementing such services would not be feasible with the existing complement of pharmacists and physicians. As a result, the infectious diseases team submitted a business case for an antimicrobial stewardship program in autumn 2007, and the proposal was subsequently approved in concept. A formal antimicrobial stewardship team (AST) was formed in July 2009, with internal pharmacy support along with 2 years of physician funding through an award from the Ministry of Health and Long-Term Care Academic Health Sciences Centre Innovation Fund.

\section{DESCRIPTION OF THE PROJECT}

The AST initially focused on collaborating with critical care staff to design and launch a prospective audit and feedback program (using evidence-based principles) and on developing an integrated antimicrobial stewardship database. 


\section{Antimicrobial Stewardship Team}

The AST initially consisted of a senior infectious diseases pharmacist, an antimicrobial stewardship pharmacy fellow, and all 5 of the hospital's staff infectious diseases physicians. Two of these physicians are also medical microbiologists, and one is active in the hospital's infection prevention and control program. Each infectious diseases physician is responsible for the AST while on call for the infectious diseases consult service, as described in more detail below.

\section{Day 3 Reflections Program}

The prospective audit and feedback program, named "Day 3 Reflections", was piloted in all 3 of the SHSC's level III ICUs: the general Critical Care Unit, with 20 beds for medical, surgical, and trauma patients; the Cardiovascular Intensive Care Unit, with 14 beds for cardiac and vascular surgery patients; and the 14-bed Ross Tilley Burn Centre, which functions as Canada's largest burn unit. These units were chosen for the pilot project because they had the highest use of broad-spectrum antimicrobials and were exempt from several of the restriction polices pertaining to use of broad-spectrum antimicrobials at SHSC. Initial activities focused on obtaining support from the critical care staff (physicians and pharmacists), promoting the "Day 3 Reflections" program, and developing electronic resources to facilitate the clinical and research activities of the team (Table 1).

Members of the AST and critical care staff worked together to develop the daily workflow of the "Day 3 Reflections" program (Figure 1). The program was designed to systematically trigger the reassessment of specific broad-spectrum antibiotics and to provide case-by-case feedback to the critical care medical and pharmacy staff. The targeted antimicrobials were a Blactam antibiotic and ß-lactamase inhibitor combination (piperacillin-tazobactam), carbapenems (ertapenem and meropenem), fluoroquinolones (ciprofloxacin and levofloxacin), third-generation cephalosporins (ceftriaxone and ceftazidime), and vancomycin. To ensure that all parties were in agreement regarding optimal antimicrobial use, the AST and critical care staff jointly developed definitions for appropriate

\section{Table 1. Goals, Activities, and Time Commitments for Prospective Audit and Feedback Program Related to Antimicrobial Stewardship}

Goals and Related Activities Obtain support from

\section{key stakeholders}

Initial meeting between AST and members of critical care medical and pharmacy staff

Creation of guidelines for appropriate use of targeted antimicrobials

Presentation at critical care grand rounds
Details of Activity

Time Commitment

Discussion of need for antimicrobial stewardship in critical care and strategies for implementation

1 meeting ( $1 \mathrm{~h})$

$20 \mathrm{~h}$ and pharmacy staff for feedback

Points of focus of presentation:

- Rationale for antimicrobial stewardship in critical care

- Proposed workflow of the "Day 3 Reflections" program

- Gathering of feedback on proposed program

Promote the "Day 3 Reflections" program
Creation of intranet page by pharmacy fellow, with assistance from institution's webmaster

Review and approval of content by all members of AST

$20 \mathrm{~h}$

Sample content of web page:

- Goals of AST

- Details of "Day 3 Reflections" program

- Supplementary educational resources

\section{Create electronic resources to \\ facilitate clinical and research activities}

Creation of Microsoft Access database

Creation of electronic progress note

Creation of electronic database linked to internal hospital databases
All relevant laboratory, microbiology, pharmacy, clinical data, suggestions, and acceptance of suggestions entered and stored in database

Assessment of impact on privacy completed as required by the privacy office

Printed from Microsoft Access database and stored as permanent part of patient's chart

Approved by Health Data Records and the Forms Committee

Web-based database created by external company on a contract basis
$70 \mathrm{~h}$ to create database and electronic progress note

Pharmacy fellow: 0.4 FTE $\times 6$ months

Senior ID pharmacist: $96 \mathrm{~h}$ ID physician: $24 \mathrm{~h}$

$\overline{\text { AST }}=$ Antimicrobial Stewardship Team, FTE $=$ full-time equivalent, ID = infectious diseases 


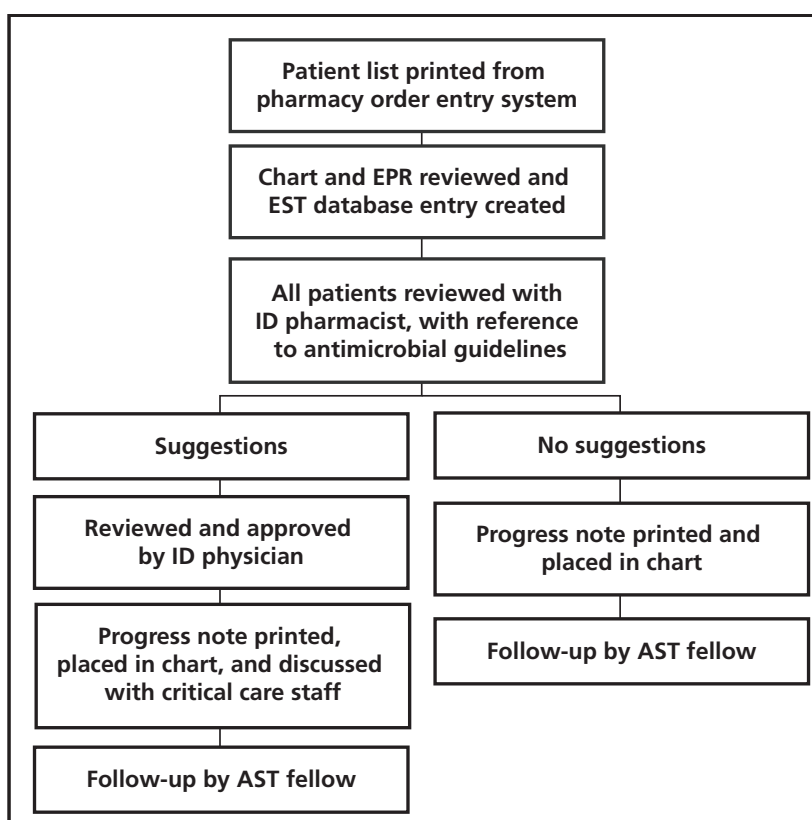

Figure 1. Daily workflow of the "Day 3 Reflections" program. AST = Antimicrobial Stewardship Team, EPR = electronic patient record, ID = infectious diseases.

and inappropriate use of each of the targeted antimicrobials before the program was launched. If, at the time of the review, a patient was receiving antimicrobials other than the targeted antimicrobials, these agents were also assessed for optimization.

The program was launched in October 2009. On regular weekdays (Monday to Friday), the antimicrobial stewardship pharmacy fellow queried the pharmacy's computer system to identify patients in the level III critical care units who were on the 3rd or 10th day of therapy with any of the targeted antimicrobials. The 3rd day of therapy was chosen in recognition that critically ill patients with signs of sepsis require timely initiation of broad-spectrum antibiotics to reduce their risk of death; however, by the 3rd day of therapy, when more information is available to assist the team in optimizing therapy (e.g., radiologic findings, culture results, laboratory values, patient's response to therapy), safe streamlining of antimicrobials is possible and rational. The second review, on the 10th day of therapy, was performed at the request of the critical care staff, to prevent excessive duration of therapy. For each patient, the bedside chart, radiologic findings, culture results, and laboratory values were reviewed, and relevant information was entered into a database. Patients were excluded from review if they were being actively followed by the infectious diseases consult service, since the physician responsible for the consult service was simultaneously responsible for reviewing patients targeted for a stewardship review. For patients who reached the 3rd or 10th day of therapy on a weekend, the review was conducted the following Monday (or Tuesday following long weekends).
The pharmacy fellow reviewed each case with the senior infectious diseases pharmacist to formulate suggestions for optimization of antimicrobial therapy according to the prespecified guidelines for appropriate use of the targeted antibiotics. These review sessions also served as education sessions for the pharmacy fellow, who thus received formal infectious diseases training. All cases in which a suggestion for optimization was made were then reviewed for approval by the infectious diseases physician on service for the AST. Once the suggestions were approved by the physician, the pharmacy fellow entered them into the database and printed computergenerated progress notes (Appendix 1, available at www.cjhponline.ca/index.php/cjhp/issue/view/85/showToc). The progress notes were then inserted into the patient's chart as part of the permanent medical record. Finally, the pharmacy fellow ensured that suggestions were discussed with the critical care staff and also followed up on each patient to determine whether the suggestions had been accepted or rejected (Figure 1). We acknowledged that the critical care staff were ultimately responsible for patient care and were therefore free to reject any suggestions without consequence.

\section{Development of Electronic Resources}

The existing databases at SHSC stored microbiology, laboratory, and pharmacy data separately. It was our goal to develop a database containing real-time, comprehensive, and integrated data for all patients admitted to the hospital. An external consulting company was contracted to collaborate with the AST and the institution's information technology (IT) department to develop a custom-made product that would serve the team's needs and that would fulfill the institution's security and compatibility requirements. The design, development, testing, and piloting phases of the database product took about 6 months. The AST was actively involved in all phases of the development, providing comprehensive feedback to the database development team. The database also underwent a mandatory review by the institution's internal privacy office to ensure that it complied with all policies pertaining to access and storage of confidential patient information. The final product, named SPIRIT (Stewardship Program Integrating Resource Information Technology), has proven to be an invaluable tool for the daily functions of the team and has the capability to support both retrospective and prospective research projects.

SPIRIT was designed to support the current and future initiatives of the AST. The database can identify patients admitted to hospital who meet any combination of several flexible criteria (e.g., location, service, date of admission, antibiotics administered, number of days of therapy, route of therapy, culture results, susceptibility results, and various other laboratory results). Manually entered data are limited to information extracted from the chart for the antimicrobial assessment. Data from microbiology, laboratory (i.e., hematology and biochemistry), pharmacy, and admission/discharge/transfer databases are 
collected in real time and are stored in tables within the database. The web-based interface of SPIRIT displays this information in a user-friendly format to facilitate assessment of antimicrobial therapy. Current antimicrobials and history of antimicrobial use are displayed separately from information about other medications, and the unique "Patient Progress Chart" view shows the patient's white blood cell counts, antibiotic use, and culture results over time, to allow for easy assessment of his or her infection history (Figure 2). The database contains sufficient information to perform drug utilization research (e.g., to determine days of therapy/1000 patient-days or defined daily doses/1000 patient-days of antibiotics used); however, the report to extract these data into a user-friendly format is still under development. As a result, the pharmacy order entry system is currently used to perform drug utilization research.

During development of the integrated database, information from each stewardship review was manually entered into a Microsoft Access database to allow for the timely launch of the
"Day 3 Reflections" program. The SPIRIT database went live in March 2010 and is now the sole database for the antimicrobial stewardship program.

\section{PROJECT EXPERIENCE}

\section{Time Commitment}

The average time spent for information-gathering by the pharmacy fellow was about $30 \mathrm{~min} /$ patient. The infectious diseases pharmacist spent about $30-45 \mathrm{~min} /$ day reviewing all cases with the pharmacy fellow and providing education to the fellow, and the infectious diseases physician spent about 10-20 $\mathrm{min} /$ day with the pharmacy fellow to review those patients for whom an antimicrobial change was recommended. From October 1, 2009, to September 30, 2010, the mean numbers of patients ( \pm standard deviation) reviewed daily were as follows: $3.0 \pm 1.7$ patients reviewed with the ID pharmacist and $1.0 \pm 0.9$ patients reviewed with the ID consult physician.

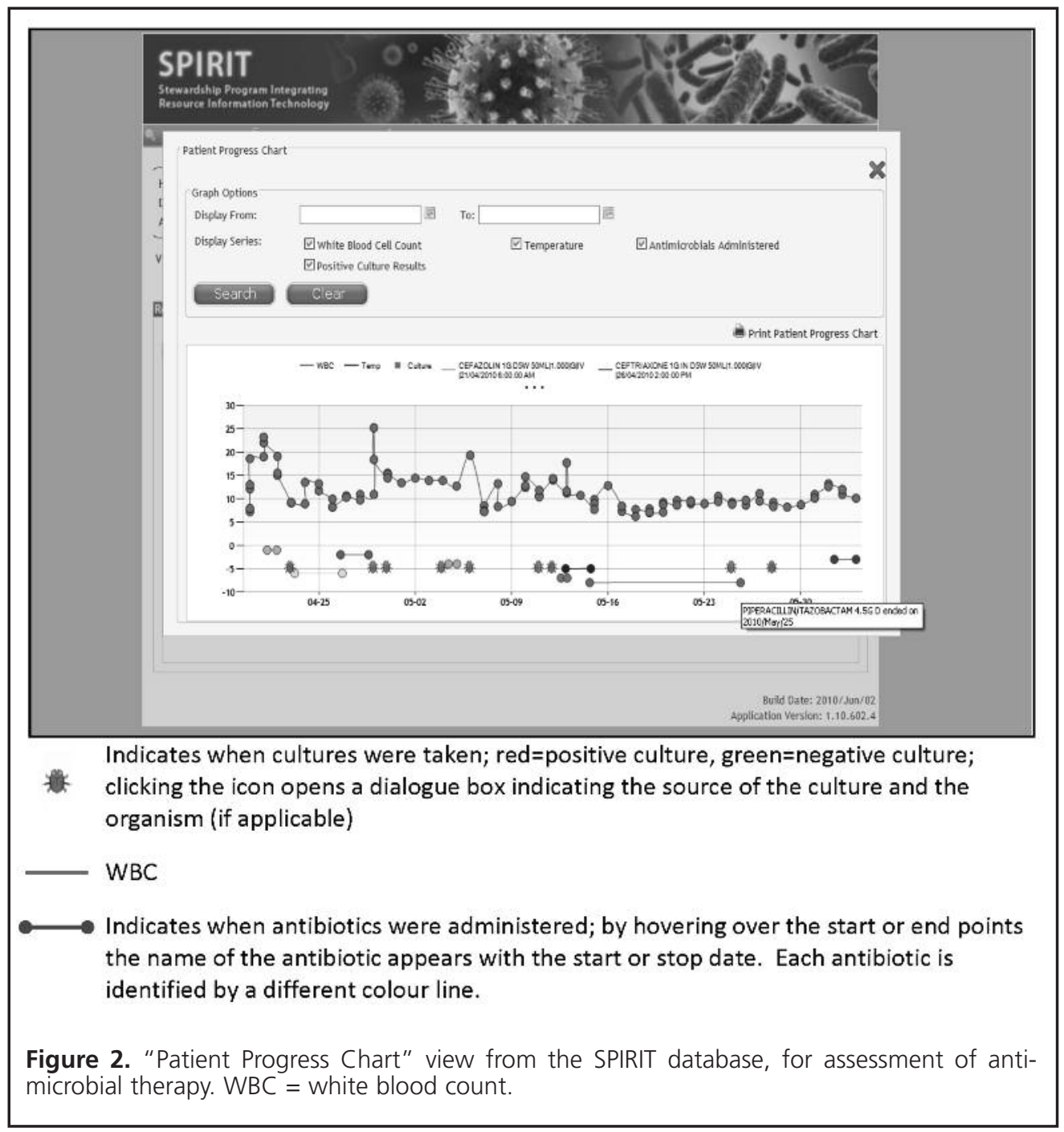




\section{Challenges and Solutions}

During implementation of the "Day 3 Reflections" program, the AST encountered several challenges. For the pharmacy members of the stewardship team, the main challenges involved communication, proper identification of patients needing an antimicrobial review, and development of electronic resources.

As described above, the program was piloted on 3 separate level III ICUs that were serviced by 4 separate teams. During the initial planning phases, it became apparent that it would not be possible to attend rounds with every team. To overcome this potential communication barrier, a unique progress note was developed to clearly indicate the AST's suggestions and rationale (see Appendix 1, available at www. cjhp-online.ca/index.php/cjhp/issue/view/85/showToc). The progress note is generated electronically and placed in the patient's bedside chart. The electronic progress note was formally reviewed and approved by the institution's Forms Committee before the program was launched. Additionally, the AST and critical care staff agreed that suggestions should be discussed with the attending physician or with another member of the critical care team who would be present at rounds in the event that the attending physician was unavailable. Initially, all suggestions were sent to liaison pharmacists for these units by e-mail. Once the liaison pharmacists gained experience, confidence, and comfort with the AST, the e-mail communication process was modified according to each pharmacist's preference. The liaison pharmacists were also encouraged to continue monitoring and altering antibiotic therapy as they had done before the intervention, with the independent review by the stewardship team being intended to provide additional infectious diseases-focused advice.

It became apparent that for a small number of patients, the planned review on day 3 of therapy was not being performed because of alterations in the original prescription (i.e., a change in dose or frequency), which resulted in an updated start date for the order (effectively producing a new day 0 in the database). As a result, some patients were reviewed later than the 3rd day of therapy, and others may have been missed altogether. Following discussions with the acute care pharmacy manager, it was determined that order entry practices could not be altered to prevent this situation. To fix the problem, it will be necessary to explore updates to the current database. At the time of writing, in late 2011, the problem remained unresolved, but it was estimated to affect less than $5 \%$ of patients.

We initially approached our institution's IT department to determine whether it would be able to develop a fully integrated database for the antimicrobial stewardship program. However, competing priorities prevented the IT department from making a commitment to complete the project in a timely manner, and we were referred to an external consulting company. The external company possessed the skill and expertise to partner with our team to build an innovative custom database compatible with existing hospital systems and satisfying all hospital privacy requirements. However, it was sometimes difficult for our team to provide all of the details needed for the various stages of design and development because of a lack of programming knowledge. To overcome this challenge, we invited a pharmacist with knowledge of computer programming to the design meetings to ensure that our needs were properly explained to the consulting company. Also, the SHSC IT department continued to provide guidance throughout the process and worked with the contract company to ensure that the final software program met all of the institution's patient security requirements. Ultimately, working with the external consulting company allowed us to overcome the database development and implementation challenges.

The infectious diseases physicians also faced some challenges related to their participation in the AST. An initial concern was that AST patient reviews might reduce the number of referrals to the infectious diseases service. This concern was alleviated by ensuring that physicians were reimbursed for time spent on AST functions and by the physicians' own realization (following implementation of the "Day 3 Reflections" program) that the AST functioned to complement and advocate for the expertise of the infectious diseases consult service. The infectious diseases physicians were also concerned that having a separate AST physician might lead to disagreements with the consulting infectious diseases physician. To circumvent this possibility, it was decided that the AST service would be covered on a rotational basis by the same physician responsible for the infectious diseases consults. On occasion, the AST physicians found it difficult to provide suggestions on cases for which they would not have the opportunity to physically assess or prospectively follow the patient. Although they did gain comfort with this process over time, it became apparent that patient care in some cases might benefit from an infectious diseases consult and follow-up. As a result, the infectious diseases physicians were given the option to recommend a formal consult. This occurs once or twice a month for patients in the level III ICUs.

\section{DISCUSSION}

The support of the critical care staff (physicians, pharmacists, and nurses) for both the antimicrobial stewardship program and the general principles of antimicrobial stewardship was a key factor in the initial success of the program. The infectious diseases physicians played a pivotal role in obtaining this support through their preliminary discussion with critical care staff (before initiation of the "Day 3 Reflections" program) and their involvement with each patient for whom a change in antimicrobial therapy was recommended. As a result, the program has been associated with substantial decreases in the 
use of broad-spectrum antibiotics and in the incidence of Clostridium difficile infections (details to be published elsewhere $)^{5}$.

Major barriers to the implementation of an antimicrobial stewardship program that have been reported in the literature include opposition from prescribers and potential antagonism of other practitioners. ${ }^{4,6}$ We were able to overcome these barriers by including critical care staff in the development of our program. Also, our collaborative, patient-centred approach, using chart notes and discussions with individual prescribers and respecting the fact that critical care staff had ultimate responsibility for and authority in the care of their patients, prevented the antagonism of our critical care colleagues. For these reasons, the AST pilot obtained the support of senior medical staff and front-line health care professionals in the critical care units at SHSC. The support of these individuals was vital to the program's success.

Formal evaluation of our stewardship program (via interrupted time series methods) will be published elsewhere. ${ }^{5}$ As a result of the year 1 successes of the AST, we recently secured ongoing hospital funding for a 1.0-FTE stewardship pharmacist, a 0.5-FTE stewardship pharmacy fellow, a 0.5-FTE stewardship infectious diseases physician, and a 0.1-FTE database analyst. Since November 1, 2010, we have expanded the scope of practice for the "Day 3 Reflections" program to medical and surgical units using a randomized, stepped-wedge clinical trial design to enable a more robust evaluation of the impact of our intervention.

\section{CONCLUSIONS}

At SHSC, the AST implemented a prospective audit and feedback program that was accepted by critical care staff. The pilot program established an acceptable workflow for all members of the team, and the development of an antimicrobial stewardship database has allowed for expansion of the program to additional medical and surgical units. The measured objective successes (i.e., reduction in days of therapy/1000 patient days and reduction in $C$. difficile infection in level III critical care units) of the AST in SHSC critical care units provided our team with the evidence needed to obtain ongoing hospital funding for the program and to expand the service to ward patients.

\section{References}

1. Dellit TH, Owens RC, McGowan JE Jr, Gerding DN, Weinstein RA, Burke JP, et al; Infectious Diseases Society of America; Society for Healthcare Epidemiology of America. Infectious Diseases Society of America and the Society for Healthcare Epidemiology of America guidelines for developing an institutional program to enhance antimicrobial stewardship. Clin Infect Dis 2007;44(2):159-177.

2. Boucher HW, Talbot GH, Bradley JS, Edwards JE, Gilbert D, Rice LB, et al. Bad bugs, no drugs: no ESKAPE! An update from the Infectious Diseases Society of America. Clin Infect Dis 2009;48(1):1-12.

3. Ontario Antimicrobial Stewardship Project: preserving antibiotics for future generations. Toronto (ON): Institute for Safe Medication Practices Canada; [cited 2010 Feb 1]. Available from: www.ismp-canada.org/abx

4. Drew RH. Antimicrobial stewardship programs: how to start and steer a successful program. J Manag Care Pharm 2009;15(2 Suppl):S18-S23.

5. Elligsen M, Walker SA, Pinto R, Simor A, Mubareka S, Rachlis A, et al. Audit and feedback to reduce broad spectrum antibiotic use in intensive care patients: a controlled interrupted time series analysis. Infect Control Hosp Epidemiol. Forthcoming.

6. Johannsson B, Beekmann SE, Srinivasan A, Hersh AL, Laxminarayan R, Polgreen PM. Improving antimicrobial stewardship: the evolution of programmatic strategies and barriers. Infect Control Hosp Epidemiol 2011;32(4):367-374.

Marion Elligsen, BScPhm, is with the Department of Pharmacy, Sunnybrook Health Sciences Centre, Toronto, Ontario.

Sandra A N Walker, BSc, BScPhm, PharmD, FCSHP, is with the Department of Pharmacy and the Division of Infectious Diseases, Sunnybrook Health Sciences Centre, and the Faculty of Pharmacy, University of Toronto, Toronto, Ontario.

Andrew Simor, MD, FRCPC, is with the Division of Infectious Diseases, Sunnybrook Health Sciences Centre, and the Department of Medicine, University of Toronto, Toronto, Ontario.

Nick Daneman, MD, MSC, FRCPC, is with the Division of Infectious Diseases, Sunnybrook Health Sciences Centre, and the Department of Medicine, University of Toronto, Toronto, Ontario.

Address correspondence to:

Dr Sandra A N Walker

Department of Pharmacy, E-302

Sunnybrook Health Sciences Centre

2075 Bayview Avenue

Toronto ON M4N 3M5

e-mail: sandra.walker@sunnybrook.ca 